International Journal of Statistics and Analysis.

ISSN 2248-9959 Volume 9, Number 1 (2019), pp. 11-23

(C) Research India Publications

https://dx.doi.org/10.37622/IJSA/9.1.2019.11-23

\title{
Socioeconomic Health Disparities in Bangladesh through Lorenz Curve and Gini Ratio: Evidence from BDHS Surveys
}

\author{
Md. Mamunur Rashid ${ }^{1}$, Tapan Kumar Roy ${ }^{2}$ and Brijesh P. Singh ${ }^{3}$ \\ ${ }^{1}$ Research Student, Department of Population Science and Human Resource \\ Development, Rajshahi University, Bangladesh \\ ${ }^{2}$ Professor, Department of Population Science and Human Resource Development, \\ Rajshahi University, Bangladesh, \\ ${ }^{3}$ Associate Professor, Department of Statistics, Banaras Hindu University, Varanasi, \\ India,
}

\begin{abstract}
Socioeconomic health disparities is a much publicized concern in developed and developing countries and recently this concept are becoming popular among demographers and health scientists in Bangladesh. Research on health disparities can be instrumental in drawing attention to the health of socioeconomically vulnerable groups in Bangladesh in the context of rapid economic growth. Although Bangladesh has already achieved noticeable progress in the field of development and health, but socioeconomic disparities of health for several markers are still noticeable. However, population within the Bangladesh experience marked differences in health and longevity due to socioeconomic disparity. In this regard, to assess the development of health sector, an attempt has been made in this study to observe the socioeconomic health disparities among women using Bangladesh Demographic and Health Survey by urban-rural and richest-poorest discrepancies. It also finds out the levels and trends of socioeconomic health disparities in Bangladesh. The study also provides urban-rural and richest-poorest disparities through Lorenz curve and Gini ratio though public health sector in Bangladesh has achieved tremendous successes over the few decades. Therefore, more public health strategies are needed for rural and poorestwomen in order to reduce these gaps further. This study gives information for policy makers about socioeconomic health disparity and will be a milestones in health sector research to take appropriate policy as well as informative to execute sustainable development goals agenda in Bangladesh.
\end{abstract}




\section{INTRODUCTION}

Health disparities have been described as "differences in health outcomes and their determinants between segments of the population, as defined by social, demographic, environmental, and geographic attributes (Penman-Aguilar et al., 2016). In recent decades, the health sector in most countries has paid attention to the issue of removing the health disparities and inequalities in health, in addition to improving the population's health (Bayatiet et al., 2017). Health disparities are the inequalities that occur in the provision of healthcare and access to healthcare across different racial, ethnic and socioeconomic groups. Health disparities are becoming widely recognized as one of the world's great challenges for the 21st century (Ruger, 2006). Health disparities are a global issue, and many different groups of people are affected by them. Although some disparities are related to economics, with poorer nations and communities receiving lower quality care, others result from discrimination (Ndiaye et al., 2008). Differences can be found according to socioeconomic status (income/education), race, ethnicity, sex or gender, sexual orientation, immigrant status, geography, and physical and mental disability. Health disparities have become of central concern in Bangladesh and globally. Populations within the Bangladesh experience marked differences in health and longevity. Health disparities frequently refer to disparities in health care, including differential access to screening and/or treatment options, or unequal availability of culturally or linguistically knowledgeable and sensitive health personnel as well as differences in access to or availability of facilities and services.

Health disparities more frequently refer to differences associated with social class and socioeconomic status (Adler et al., 2010). Socio-economic inequalities in health care in Bangladesh come near the top of international league tables of equity in health care financing. Benefit incidence studies show that public health care expenditure in Bangladesh disproportionately benefits poorer people. However, this does not necessarily mean that health care is distributed fairly in relation to need, since poorer people tend to be sicker and so have greater need for health care (Cookson et al., 2016). Lower socioeconomic status is associated with higher mortality, and the greatest disparities occur in middle adulthood. Disparities in the quality of health care delivered to different socioeconomic groups have been noted in studies. Compared to disparities in health status, which are caused by a variety of social, economic, environmental and other factors, disparities in the quality of health care services are considered more amenable to changes in the health care delivery system (Hussey et al., 2008). There is substantial evidence regarding racial/ethnic and socioeconomic disparities in health-related behaviors, health status, and health outcomes (O'Keefe et al., 2015). In this context, an attempt has been made in this study to know the socioeconomic health disparities in Bangladesh using Lorenz curve and Gini concentration ratio. 


\section{DATA AND METHODS}

This study is based on the extensive analysis of seven comparable data sets from the BDHS carried out in 1993-94, 1996-97, 1999-2000, 2004, 2007, 2011 and 2014. In this analysis, women who had most recent child have been considered for the above surveys. The selected some socioeconomic health indicators such as antenatal care, healthcare facility for recent child, ideal number of children, age at marriage, underweight and overweight have been considered.In this study, Lorenz curve has been used to measure socioeconomic health disparities. Generally, Lorenz curve is introduced as a graphic device for representing the inequality or disparity of two distributions and is illustrated by plotting the cumulative percentage of the number of areas $\left(\mathrm{Y}_{\mathrm{i}}\right)$ against the cumulative percentage of population $\left(\mathrm{X}_{\mathrm{i}}\right)$ of localities in a country. The perfectly distributed population in a country means the cumulative share of population would be equal to the cumulative share of localities. Such equality of distribution is represented by a diagonal line. This diagonal line is compared to the actual distribution and the gap between the ideal and actual lines is interpreted as the degree of inequality. The Gini coefficient is a number between 0 and 1, where 0 corresponds with perfect equality (where everyone has the same income) and 1 corresponds with perfect inequality. The BDHS constructed cumulative percentage wealth score is considered in $\mathrm{X}$-axis and cumulative proportion of several health indicators (age at marriage, ideal number of children, adequate antenatal care visits for most recent child, healthcare facility for recent child, underweight and overweight) are plotted in Y-axis.

\section{FINDINGS}

\section{Levels and Trends of Socioeconomic Health Disparities in Bangladesh}

In this study, some socioeconomic public health indicators such as age at marriage, ideal number of children, adequate antenatal care visits for most recent child, healthcare facility for recent child, underweight and overweight have been considered from Bangladesh Demographic and Health Survey (BDHS). Here, seven round BDHS survey data have been used to know the levels and trends of disparities. The trends of disparities among public health indicators by socioeconomic status in Bangladesh during 1993 to 2014 have been observed.

Figure 1 shows that age at marriage is increasing day by day due to socioeconomic development for Bangladesh. In richest group, age at marriage was 22.1 years in 1993-94 but every BDHS survey it increased on average three or four years up to 2011. However, age at marriage increased from 37.3 years in 2011 to 39.2 years in 2014 approximately two years. The similarly pattern was observed poorest group. The age at marriage dramatically has increased approximately eight years from 2011 to 2014. 


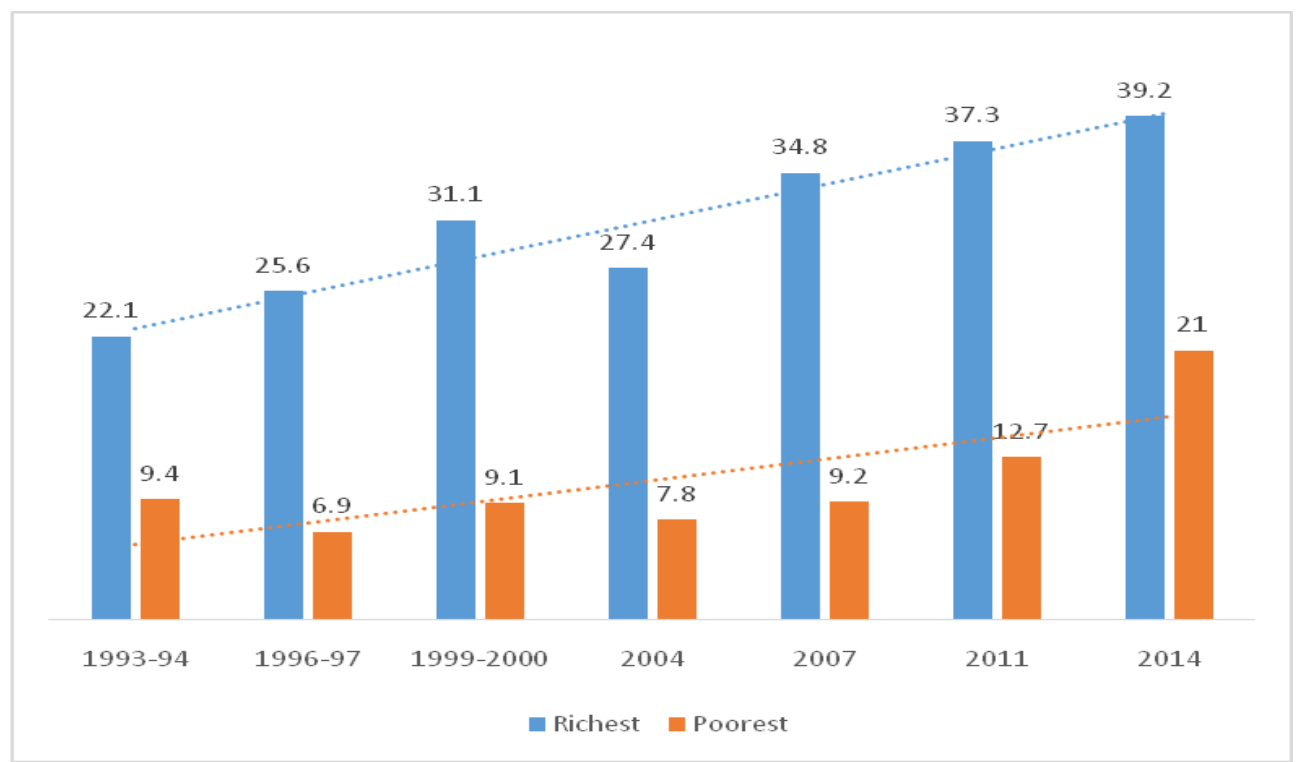

Figure 1: Trends of richest-poorest disparity in case of age at marriage in Bangladesh during 1993 to 2014

Figure 2 shows that ideal number of children is increasing day by day due to socioeconomic development for Bangladesh. In richest group, ideal number of children was 72.0 years in 1993-94 but every BDHS survey it increased on average one or two years up to 2004 . However, ideal number of children was stable from 86.3 years in 2011 to 86.3 years in 2014 . The similarly pattern was observed in poorest group. The ideal number of children dramatically has increased approximately nine years from 2007 to 2011 .

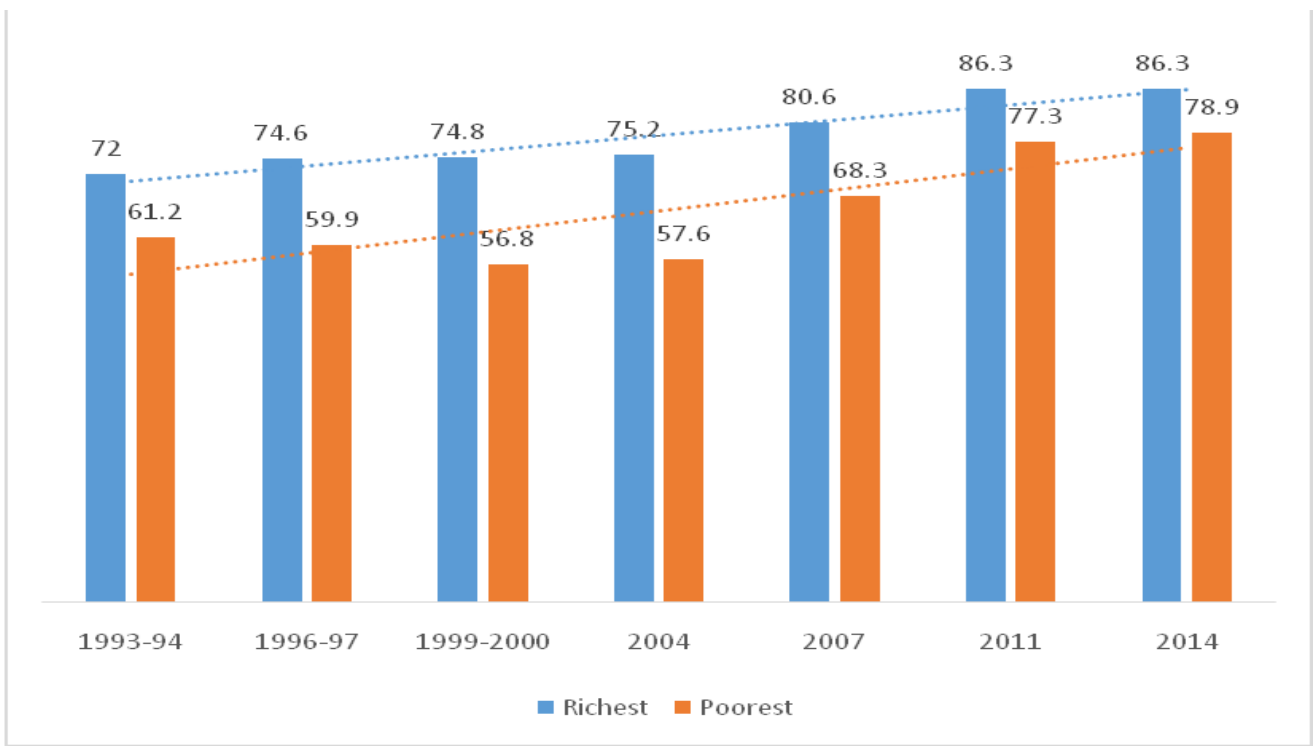

Figure 2: Trends of richest-poorest disparity in case of ideal number of childrenin Bangladesh during 1993 to 2014 
Figure 3 shows that antenatal care is increasing day by day due to socioeconomic development for Bangladesh. In richest group, antenatal care was 25.4 years in 199394 but every BDHS survey it increased on average four or five years up to 2011 . However, antenatal care decreased from 53.1 years in 2011 to 48.0 years in 2014 approximately five years. The similarly pattern was observed poorest group. The age at marriage dramatically has increased approximately twelve years from 2011 to 2014.

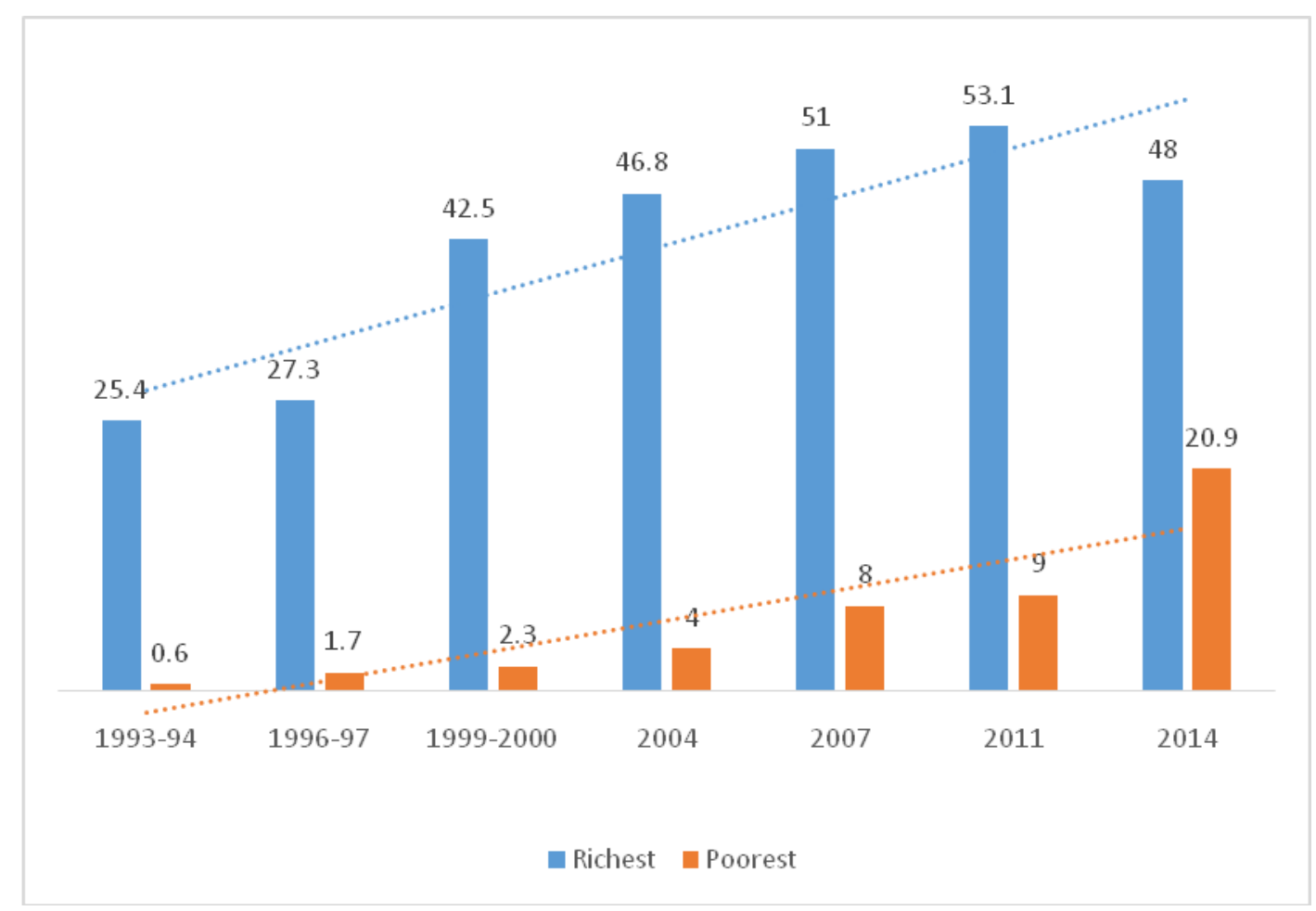

Figure 3: Trends of richest-poorest disparity in case of adequate antenatal care for recent childin Bangladesh during 1993 to 2014

Figure 4 shows that healthcare facility for recent child is increasing day by day due to socioeconomic development for Bangladesh. In richest group, healthcare facility for recent child was 18.2 years in 1993-94 but every BDHS survey it increased on average three years up to 1996-97. However, healthcare facility for recent child increased from 57.6 years in 2011 to 81.3 years in 2014 approximately twenty two years. The similarly pattern was observed poorest group. The healthcare facility for recent child dramatically has increased approximately forty five years from 2011 to 2014. 


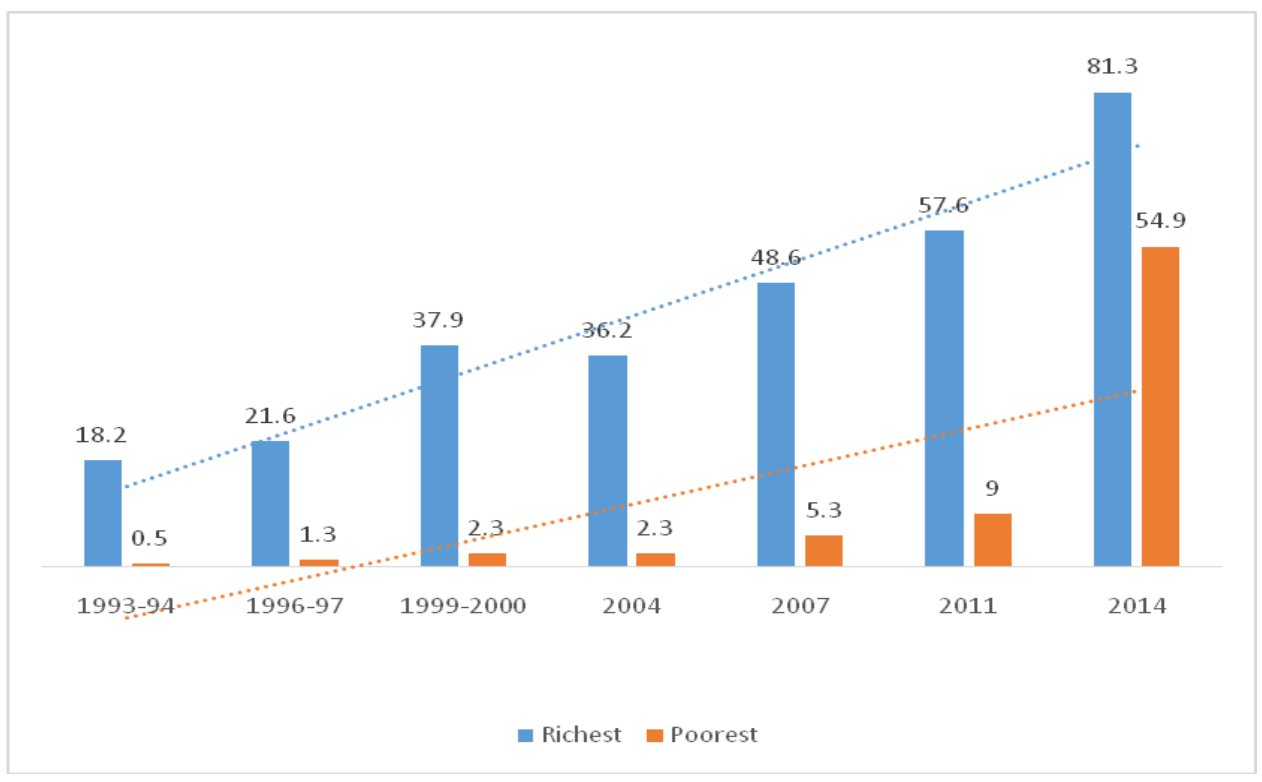

Figure 4: Trends of richest-poorest disparity in case of healthcare facility for recent child in Bangladesh during 1993 to 2014

Figure 5 shows that underweight is decreasing day by day due to socioeconomic development for Bangladesh. In richest group, underweight was 60.5 years in 1996-97 but every BDHS survey it decreased on average four or five years up to 2011. However, underweight decreased from 38.8 years in 2011 to 29.8 years in 2014 approximately nine years. The similarly pattern was observed poorest group. The underweight dramatically has increased approximately four years from 2011 to 2014 .

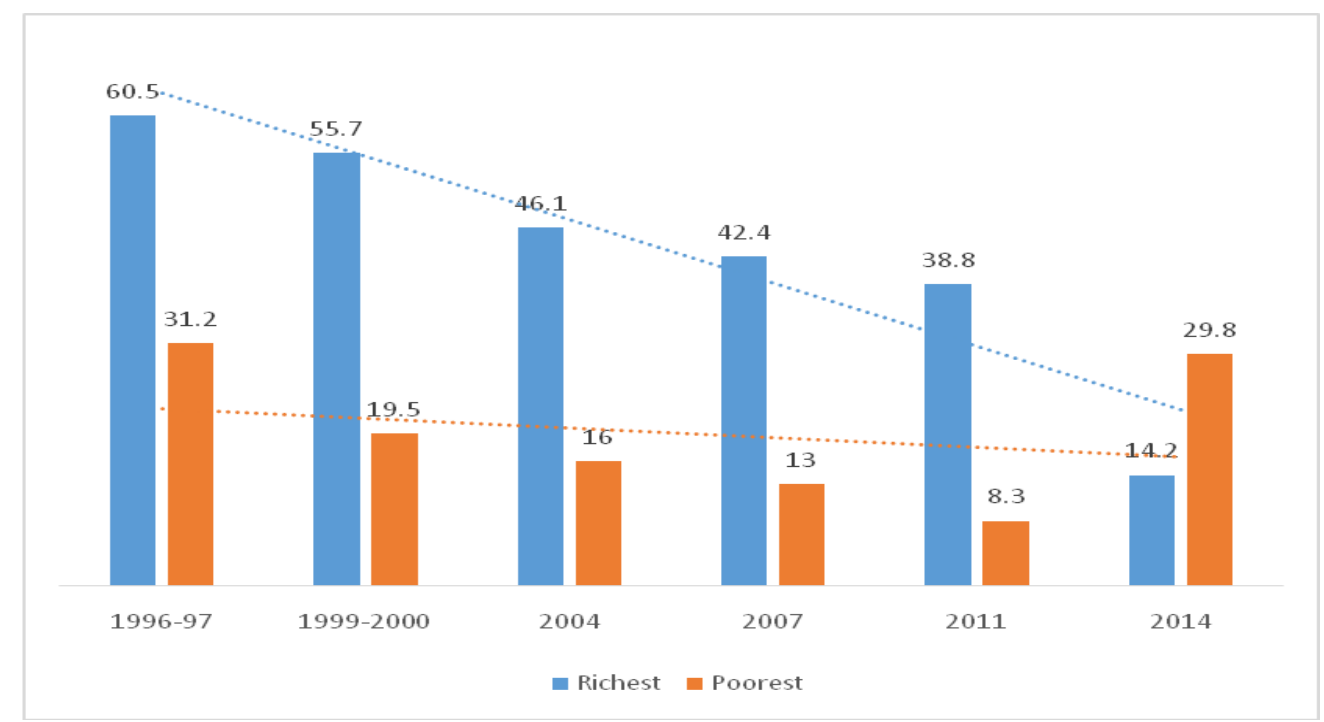

Figure 5: Trends of richest-poorest disparity in case of underweightin Bangladesh during 1993 to 2014 
Figure 6 shows that overweight is increasing day by day due to socioeconomic development for Bangladesh. In richest group, overweight was 11.3 years in 1996-97 but every BDHS survey it increased on average six or seven years up to 2007. However, overweight decreased from 36.3 years in 2011 to 26.8 years in 2014 approximately ten years. The similarly pattern was observed poorest group. The overweight dramatically has increased approximately four years from 2011 to 2014 .

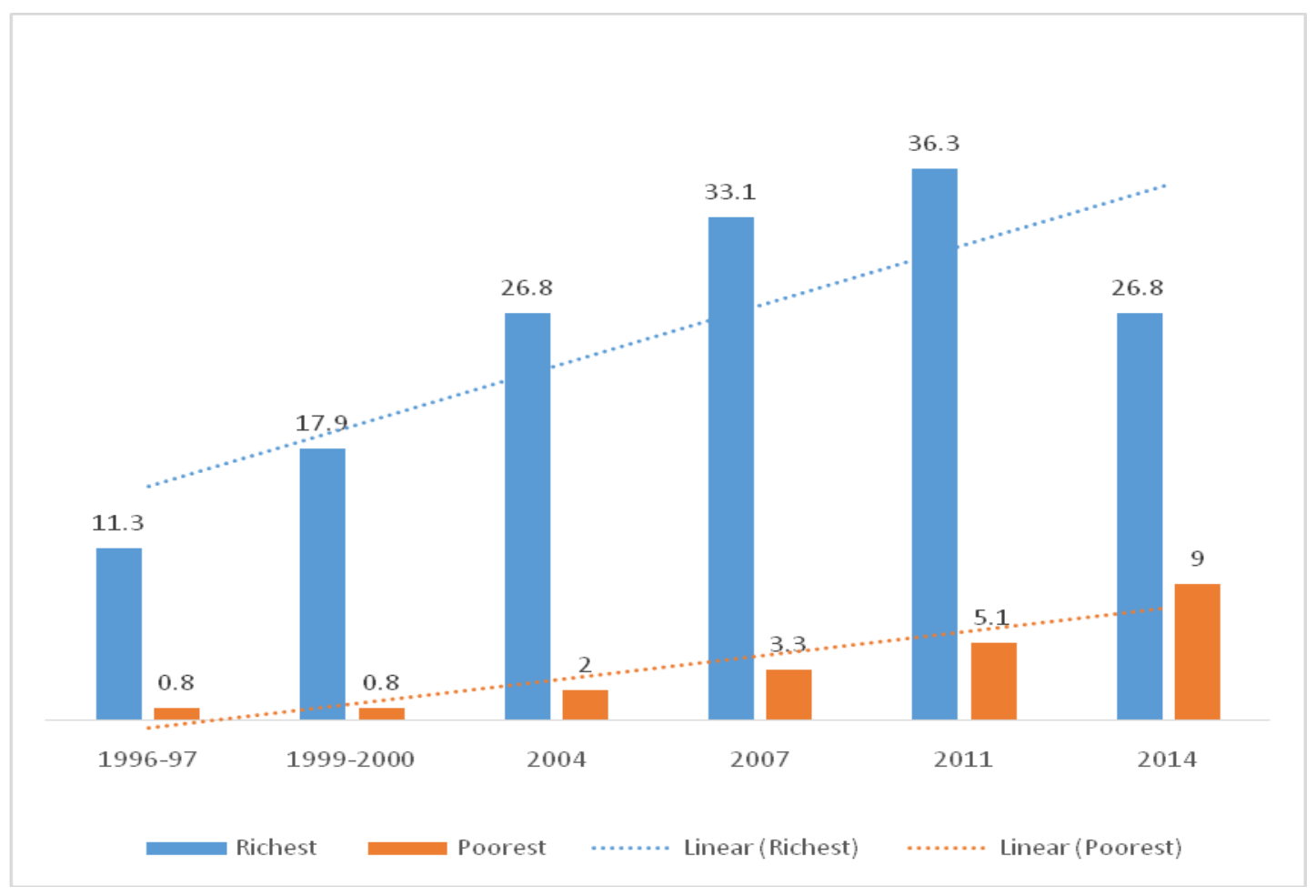

Figure 6: Trends of richest-poorest disparity in case of overweightin Bangladesh during 1993 to 2014

\section{Differentials of Socioeconomic Health Disparities in Bangladesh}

In this section, the differentials of socioeconomic health disparities and their significance have been observed by using chi-square p-value. Socioeconomic status (SES) explains the rural/urban difference in ANC quality, and not the reverse, reducessupport for the hypotheses that high socioeconomic background women receive better quality of care because of where they live. This does not mean the quality of care in one's place of residence is unimportant, it means a woman's SES, if high, may enable her to obtain better quality of care above what is available in her immediate community.Women of higher SES are not limited to seeking care where they live, they can go outside of their communities to access higher quality care. The urban-rural, richest-poorest and literate-illiterate comparison based on Bangladesh Demographic and Health Survey (BDHS) 2014 are shown in this section. 
Table 1: Disparities of health indicators by urban-rural according to BDHS 2014

\begin{tabular}{|l|c|c|c|c|}
\hline Indicator & Urban (\%) & Rural (\%) & $\begin{array}{c}\text { Urban/Rural } \\
\text { ratio }\end{array}$ & p-value \\
\hline Age at marriage & 34.9 & 25.4 & 1.37 & 0.000 \\
\hline Ideal number of children & 86.5 & 79.8 & 1.08 & 0.000 \\
\hline $\begin{array}{l}\text { Adequate ANC visits for } \\
\text { recent child }\end{array}$ & 45.8 & 25.4 & 1.80 & 0.000 \\
\hline $\begin{array}{l}\text { Healthcare facility for the } \\
\text { recent child }\end{array}$ & 79.0 & 59.4 & 1.32 & 0.000 \\
\hline Underweight & 17.2 & 26.3 & 0.65 & 0.000 \\
\hline Overweight & 25.0 & 12.1 & 2.06 & 0.000 \\
\hline
\end{tabular}

The levels of disparities of different health indicators differed significantly with urban-rural groups. From above table, we see that urban pregnant women receive adequate antenatal care service more often (45.8\%) than rural women $(25.4 \%)$. Similarly, significantly the levels of age at marriage, ideal number of children, health care facility for recent child and underweight in the rural groups revealed their greater level of vulnerability than the urban groups. The rural groups only revealed a better situation in terms of being overweight, it was significantly lower in these groups.

In this study, the urban/rural ratios for these indicators are also calculated. In general, a ratio equal to one indicates no disparity, which means the greater the deviation from the ratio one, the greater is the disparity. For all the indicators except being underweight, urban/rural ratios greater than one indicate a better public health situation in the urban groups as compared to their counterparts. In contrast, for the overweight indicator a ratio greater than one indicates a worse situation in the urban groups.Based on the urban/rural ratio, higher disparities (deviation from 1.0) were found for the received adequate ANC visits for the most recent child (ratio=1.80), overweight $($ ratio $=2.06)$, healthcare facility for recent child (ratio=1.32) and age at marriage (ratio=1.37). For the underweight indicator the ratio was 0.65 , which also indicated lower disparities. Comparatively, disparities were stronger rural groups than urban groups. Disparities for ideal number of children indicators were lowest for both equity makers. 
Table 2: Disparities of health indicators by richest-poorest according to BDHS 2014

\begin{tabular}{|l|c|c|c|c|}
\hline Indicator & Richest (\%) & Poorest (\%) & $\begin{array}{c}\text { Richest/Poorest } \\
\text { ratio }\end{array}$ & p-value \\
\hline Age at marriage & 39.2 & 21.0 & 1.86 & 0.000 \\
\hline $\begin{array}{l}\text { Ideal number of } \\
\text { children }\end{array}$ & 86.3 & 78.9 & 1.09 & 0.000 \\
\hline $\begin{array}{l}\text { Adequate ANC visits } \\
\text { for recent child }\end{array}$ & 48.0 & 20.9 & 2.29 & 0.000 \\
\hline $\begin{array}{l}\text { Healthcare facility for } \\
\text { recent child }\end{array}$ & 81.3 & 54.9 & 1.48 & 0.000 \\
\hline Underweight & 14.2 & 29.8 & 0.47 & 0.000 \\
\hline Overweight & 26.8 & 9.0 & 2.97 & 0.000 \\
\hline
\end{tabular}

The levels of disparities of different public health indicators differed significantly with richest-poorest groups. From above table, we see that richest pregnant women received adequate antenatal care service more often $(48.0 \%)$ than poorest women $(20.9 \%)$. Similarly, significantly the levels of age at marriage, ideal number of children, health care facility for recent child and underweight in the poorest groups revealed their greater level of vulnerability than the urban groups. The poorest groups only revealed a better situation in terms of being overweight, it was significantly lower in these groups.

In this study the richest/poorest ratios for these indicators are also calculated. In general, a ratio equal to one indicates no disparity, which means the greater the deviation from the ratio one, the greater is the disparity.For all the indicators except being underweight, richest/poorest ratios greater than one indicate a better public health situation in the richest groups as compared to their counterparts. In contrast, for the overweight indicator a ratio greater than one indicates a worse situation in the richest groups.

Based on the richest/poorest ratio, higher disparities (deviation from 1.0) were found for adequate ANC visits for the most recent child (ratio=2.29), overweight $($ ratio $=2.97)$, healthcare facility for the most recent child (ratio=1.48), ideal number of children $($ ratio $=1.09)$ and age at marriage $(r a t i o=1.86)$. For the underweight indicator the ratio was 0.47 , which also indicated lower disparities.

Comparatively, disparities were stronger poorest groups than richest groups. Disparities for ideal number of children indicators were lowest for both equity makers. 


\section{Measurement of Socioeconomic Health Disparities Using Lorenz Curve and Gini Ratio}

In this section, Lorenz curve and Gini Concentration ratio have been applied to BDHS 2014 data to measure socioeconomic inequalities or disparities among women in Bangladesh.The following figure represents the concentration curve of antenatal care where the curves lies below the diagonal line. The value of Gini's coefficient is 0.31 which means high inequality in antenatal care across the wealth of population.

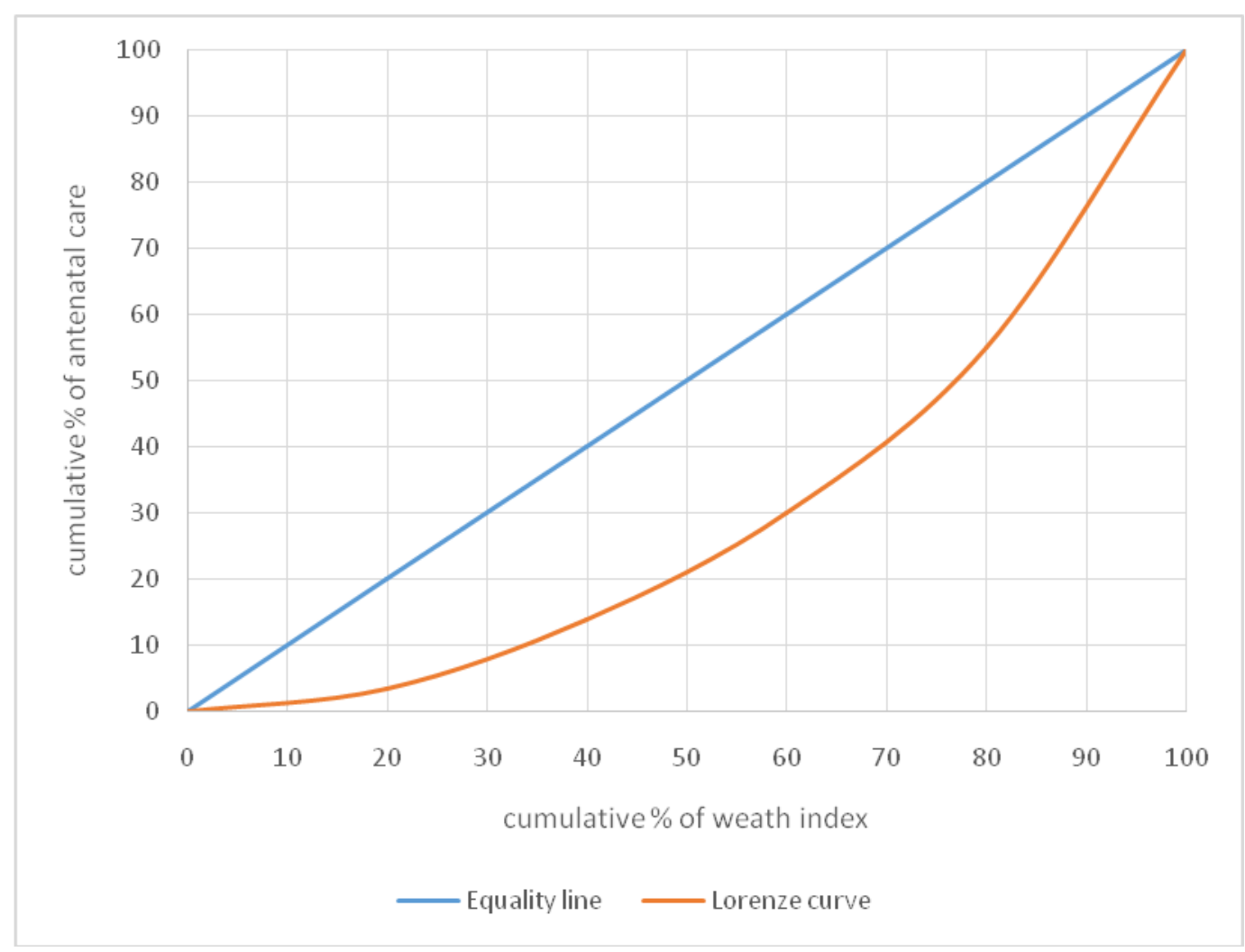

Figure 7: Lorenz curve of concentration for antenatal care

The following figure represents the concentration curve of delivered health care facility where the curves lies below the diagonal line. The value of Gini's coefficient is 0.11 which means less inequality in delivered health care facility across the wealth of population. 


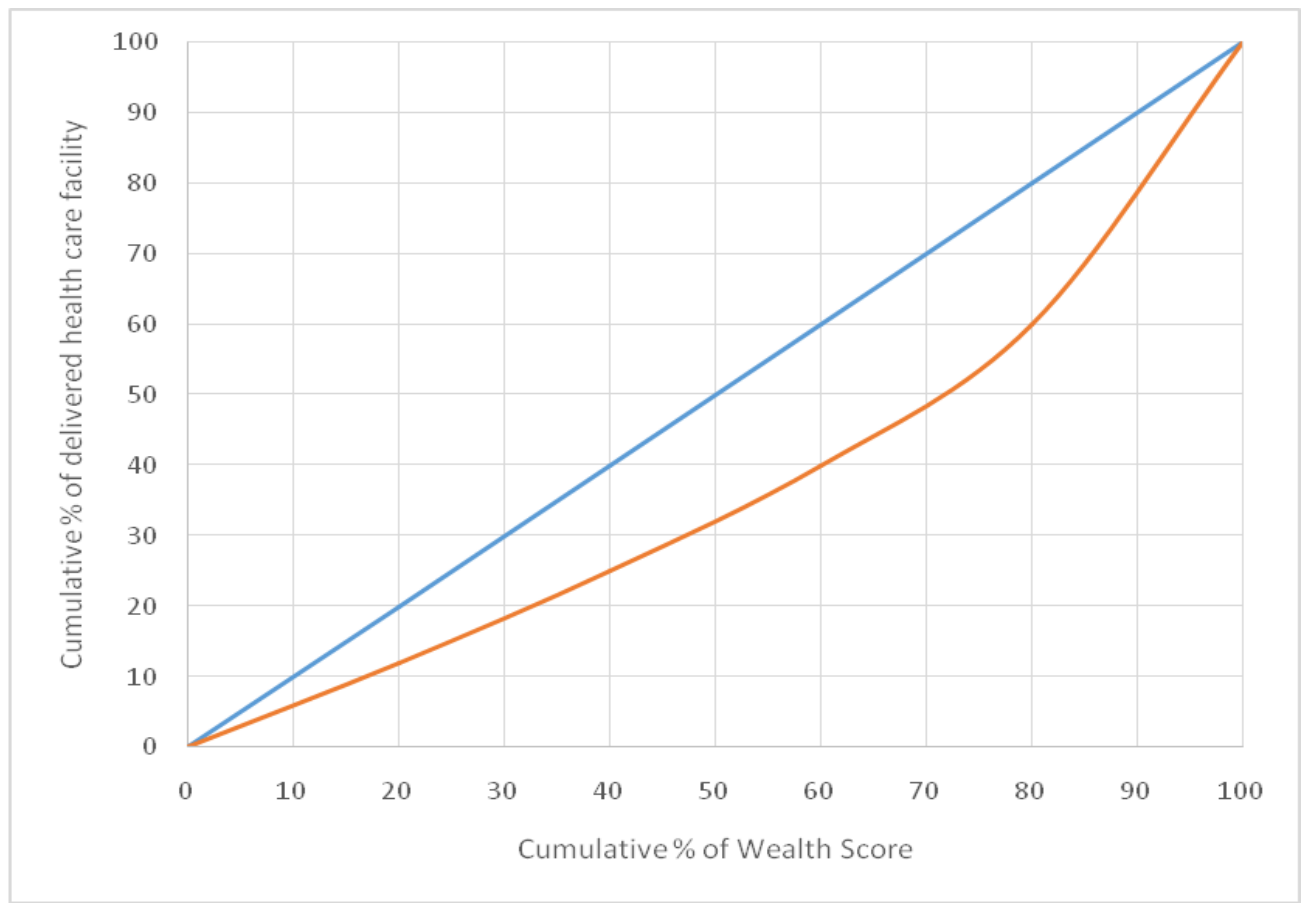

Figure 8: Lorenz Curve of Concentration for delivered health care facility

The following figure represents the concentration curve of age at first marriage where the curves lies below the diagonal line. The value of Gini's coefficient is 0.018 which means less inequality in age at first marriage across the wealth of population.

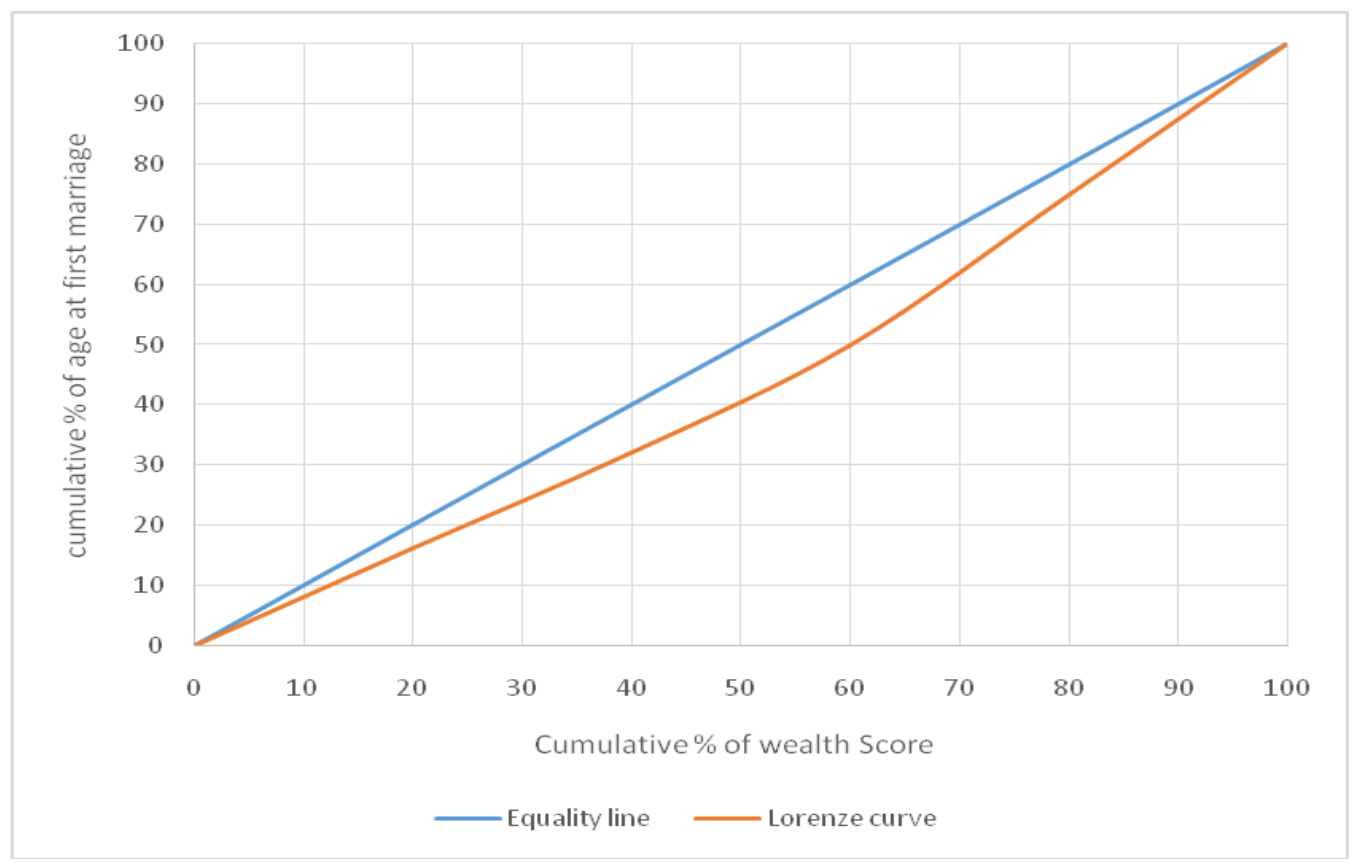

Figure 9: Lorenz Curve of Concentration for age at first marriage 


\section{DISCUSSION AND CONCLUSIONS}

In this study, the long-term trends for socioeconomic disparity and some health related indicators in Bangladesh shows interesting findings that are important for policymakers and stakeholders. One of the important findings is the increasing tendency of health indicators (except being underweight) over time, which delivers the message that socioeconomic development occur in Bangladesh. Despite much progress, social economic disparities persist in Bangladesh as well as wealth and power are highly concentrated and the poorer and more disadvantaged segments of the population reap few of the benefits of development, yet relevant public policies to redress the situation are rarely implemented. Poverty is higher in rural areas than in urban areas, the health system caters to the rich, urban elite and the poor have less access to health care than the rich (Khan et al., 2011).If the poor are relatively deprived of effective health care interventions, income-related disparities in health will be exacerbated. Not only is this of immediate concern, it will strengthen the health-poverty trap that can retard economic growth (Doorslaer et al., 2008). The dominant schools of thought on health disparities in both developed and low-income countries are the structural and the behavioral, the structural school maintains that disparities arise as a result of differences in the socio-economic structure of society which lead to poverty and its health consequences, the behavioral school holds that cultural and behavioral patterns associated with and contributing to poverty explain much of the observed difference in health outcomes (Macassa et al., 2006). The socioeconomic disparities in health will be biased if the reporting of health varies directly with the socio-economic characteristic and/or with demographic factors correlated with that characteristic (Bago d'Uva et al., 2008). It is therefore important to understand how wider changes in socioeconomic conditions impact on health disparities in order to shape the design of an effective set of public policies to tackle the issue (Allanson et al., 2013).

There is a very significant difference in health disparities between richest and poorest population in Bangladesh. The health disparities varies richest-poorest group in Bangladesh although the inequality prevalence has been decreased in the country as a whole. The overall condition of health disparities in richest group is better than poorest group. This study showed that the richest population are more than free from urban-rural and richest-poorest health disparities than poorest populations. Therefore, Bangladesh government should pay more attention to eliminate the inequalities in the economy as well as health care distribution richest-poorest group.

\section{REFERENCES}

Adler, N. E., \& Stewart, J. (2010). Health disparities across the lifespan: meaning, methods, and mechanisms. Annals of the New York Academy of Sciences, 1186, 5-23. https://doi.org/10.1111/j.1749-6632.2009.05337.x

Allanson, P., \& Petrie, D. (2013). Longitudinal methods to investigate the role of health determinants in the dynamics of income-related health inequality. Journal of 
Health Economics, 32(5), 922-937. https://doi.org/10.1016/j.jhealeco.2013.07.001

Bago d'Uva, T., Van Doorslaer, E., Lindeboom, M., \& O'Donnell, O. (2008). Does reporting heterogeneity bias the measurement of health disparities? Health Economics, 17(3), 351-375. https://doi.org/10.1002/hec.1269

Bayati, M., Feyzabadi, V. Y., \& Rashidian, A. (2017). Geographical Disparities in the Health of Iranian Women: Health Outcomes, Behaviors and Health-care Access Indicators. International Journal of Preventive Medicine, 8, 11. https://doi.org/10.4103/ijpvm.IJPVM_67_16

Cookson, R., Propper, C., Asaria, M., \& Raine, R. (2016). Socio-Economic Inequalities in Health Care in England. Fiscal Studies, 37(3-4), 371-403. https://doi.org/10.1111/j.1475-5890.2016.12109

Doorslaer, E., \& O'Donnell, O. (2008). Measurement and Explanation of Inequality in Health and Health Care in Low-Income Settings.

Hussey, P., Anderson, G., Berthelot, J.-M., Feek, C., Kelley, E., Osborn, R., Epstein, A. (2008). Trends in socioeconomic disparities in health care quality in four countries. International Journal for Quality in Health Care, 20(1), 53-61. https://doi.org/10.1093/intqhe/mzm055

Khan, M. M. H., Krämer, A., Khandoker, A., Prüfer-Krämer, L., \& Islam, A. (2011). Trends in sociodemographic and health-related indicators in Bangladesh, 1993-2007: will inequities persist? Bulletin of the World Health Organization, 89(8), 583-593. https://doi.org/10.2471/BLT.11.087429

Macassa, G., \& Burström, B. (2006). Determinants of social inequalities in child mortality in Mozambique: what do we know? What could be done? African Journal of Health Sciences, 13(1-2), 139-143.

Ndiaye, K., Krieger, J. R., Warren, J. R., Hecht, M. L., \& Okuyemi, K. (2008). Health Disparities and Discrimination: Three Perspectives. Journal of Health Disparities Research and Practice, 2(3), 51-71. https://doi.org/10.1901/jaba.2008.2-51

O'Keefe, E. B., Meltzer, J. P., \& Bethea, T. N. (2015). Health Disparities and Cancer: Racial Disparities in Cancer Mortality in the United States, 2000-2010. Frontiers in Public Health, 3. https://doi.org/10.3389/fpubh.2015.00051

Penman-Aguilar, A., Talih, M., Huang, D., Moonesinghe, R., Bouye, K., \& Beckles, G. (2016). Measurement of Health Disparities, Health Inequities, and Social Determinants of Health to Support the Advancement of Health Equity. Journal of Public Health Management and Practice: JPHMP, 22 Suppl 1, S33-S42. https://doi.org/10.1097/PHH.0000000000000373

Ruger, J. P. (2006). Ethics and governance of global health inequalities. Journal of Epidemiology \& Community Health, 60(11), 998-1002. https://doi.org/10.1136/jech.2005.041947 
